\title{
MicroRNA-193b regulates human ovarian cancer cell growth via targeting STMN1
}

\author{
HAIYAN LI, YUPING XU and DANNI ZHAO
}

\begin{abstract}
Department of Gynaecology, Shi Jia Zhuang The Third Hospital, Shijiazhuang, Hebei 050011, P.R. China
\end{abstract}
Received May 2, 2019; Accepted January 13, 2020

DOI: $10.3892 /$ etm.2020.9033

\begin{abstract}
Ovarian cancer is the eighth most common malignancy among women worldwide. Ovarian cancer exhibits no obvious symptoms in the early stage of tumorigenesis and currently, no effective methods for the early detection and treatment of ovarian cancer have been established. Therefore, the identification of novel targets is critical to the early diagnosis and clinical treatment of ovarian cancer. microRNAs (miRs) are small non-coding RNAs, which serve an important biological role in a number of physiological processes and in oncogenesis. Previous studies have reported that miRNA-193b is dysregulated in a variety of types of human cancer. However, the roles of miRNA-193b in human ovarian cancer has not been determined. The present study investigated the roles of miRNA-193b in human ovarian cancer cells. Reverse transcription-quantitative PCR results indicated that the expression of miRNA-193b in ovarian cancer cells was significantly down-regulated compared with non-malignant cells. Cell counting kit- 8 results indicated that the up-regulation of miRNA-193b inhibited ovarian cancer cell proliferation and induced ovarian cancer cell apoptosis. The present study also indicated that stathmin 1 (STMN1) was a direct target of miRNA-193b, and the up-regulation of miRNA-193b significantly decreased the expression of STMN1 in ovarian cancer cells. In conclusion, the results demonstrated that miRNA-193b serves as a tumor suppressor in human ovarian cancer by inhibiting cell proliferation and inducing cell apoptosis. Therefore, the assessment of miRNA-193b may provide insight into a novel diagnostic biomarker and potential therapeutic target for patients with ovarian cancer.
\end{abstract}

\section{Introduction}

Ovarian cancer is the eighth most common malignancy among women worldwide, and is also the eighth leading cause of

Correspondence to: Dr Haiyan Li, Department of Gynaecology, Shi Jia Zhuang The Third Hospital, 15 Tiyu South Street, Shijiazhuang, Hebei 050011, P.R. China

E-mail: alex_hyl@yeah.net

Key words: ovarian cancer, microRNA-193b, proliferation, apoptosis, stathmin 1 cancer associated mortality in women (1). A previous study has indicated $\sim 295,414$ new patients with ovarian cancer patients were diagnosed and $\sim 184,799$ women die due to ovarian cancer every year (1). The high mortality that accompanies ovarian cancer is due to lack of obvious symptoms and effective methods for the early detection of the disease (2). The current treatments for ovarian cancer are surgery and chemotherapy, but the prognosis of ovarian cancer is poor and the 5 -year survival rate is only $\sim 44 \%$ (1). Therefore, the investigation into mechanisms underlying the pathogenesis of ovarian cancer tumorigenesis and the development of novel and effective methods for the treatment of patients with ovarian cancer patients are urgently required.

microRNAs (miRNAs) are a series of small, conserved on-coding short RNAs, and miRNAs are formed by 18 to 25 nucleotides in length (3). Previous studies have indicated that miRNAs are dysregulated in a number of human cancers, and regulated the target gene expression by binding to the 3' untranslated region (UTR) of target messenger RNAs (mRNAs). Therefore, miRNAs have been suggested to regulate human cancer cell growth and differentiation (4-8). A previous study has also indicated that miRNAs can regulate human tumorigenesis (9). In a variety of human tumor types, distinct miRNA expression profiles have been identified, which have been associated with tumor histological subtypes (10). Therefore, miRNAs can be used as biomarkers for the diagnosis and prognosis of a number of cancer types. A growing body of evidence has suggested that miR-193b is significantly down-regulated compared with non-cancerous tissues in a number of human cancer types, including: Liver cancer, lung cancer, pancreatic cancer, esophageal squamous cell carcinoma, cervical cancer and ovarian cancer (11-15). Studies have also demonstrated that miR-193b played as a tumor-suppressive miRNA in human cancer by regulating cancer cell proliferation, migration, invasion and metastasis (11-15). Although these studies reported that the expression of miR-193b is altered in a number of cancers, including in ovarian cancer, the roles of miR-193b in ovarian cancer have not, to the best of our knowledge, yet been fully determined.

The current study investigated the biological effects of miR-193b in ovarian cancer cells, and studied the underlying molecular mechanisms. The results demonstrated that miR-193b was down-regulated in ovarian cancer cells and regulated ovarian cancer cell proliferation and apoptosis by targeting the stathmin 1 (STMN1) gene. These findings 
provide insights into the development of potential diagnostic and therapeutic tools for use in the treatment of ovarian cancer in the future.

\section{Materials and methods}

Cells culture. A2780cp, A2780s, SKOV3 and CAOV3 cells were purchased from the American Type Culture Collection. Human ovarian epithelium cell line HOEC was also purchased from ATCC. All cells were cultured in Dulbecco's modified Eagle's medium (DMEM; Invitrogen; Thermo Fisher Scientific, Inc.) supplemented with $10 \%$ fetal bovine serum (FBS; Invitrogen; Thermo Fisher Scientific, Inc.), $1 \%$ penicillin (Invitrogen; Thermo Fisher Scientific, Inc.) and $1 \%$ streptomycin (Invitrogen; Thermo Fisher Scientific, Inc.) in a humidified incubator with $5 \% \mathrm{CO}_{2}$ at $37^{\circ} \mathrm{C}$.

Transfection. miR-negative control (NC), miR-193b mimic, miR-193b inhibitor and inhibitor control were purchased from Ambion; Thermo Fisher Scientific, Inc. The miR-193b mimic or inhibitor were diluted in Opti-MEM medium (Invitrogen; Thermo Fisher Scientific, Inc.) at room temperature (RT) for $15 \mathrm{~min}$. A2780cp, A2780s and CAOV3 cells were transfected with miR-NC or miR-193b, while HOEC cells were transfected with miR-193b inhibitor or inhibitor control and cultured for 48 h. Transfection was performed using Lipofectamine ${ }^{\circledR} 3000$ (Thermo Fisher Scientific, Inc) according to the manufacturer's recommendations.

Cell viability detection assay. The Cell Counting Kit-8 (CCK-8) assay (Beyotime Institute of Biotechnology) was performed to measure cell viability according to the manufacturer's recommendations. Mock or transfected cells were seeded and cultured. Subsequently, $10 \mu 1 \mathrm{CCK}-8$ was added into the cell culture medium and incubated at $37^{\circ} \mathrm{C}$ for an additional $4 \mathrm{~h}$. Subsequently, absorbance was measured at $450 \mathrm{~nm}$ using a plate reader (Molecular Devices, Spectramax).

Cell apoptosis assay. Mock or transfected cells were seeded and cultured. Ovarian cancer cell apoptosis was measured using the Apo-ONE Homogeneous Caspase-3/7 Assay kit (Promega Corporation) according to the manufacturer's instructions. CellTiter-Blue (Promega Corporation) was used to measure cell number. The relative Caspase-3/7 activity was calculated using the ratio of Apo-ONE and CellTiter-Blue signals.

RNA extraction and reverse transcription-quantitative $(R T-q) P C R$. Total RNA was isolated from mock or transfected ovarian cancer cells using TRIzol reagent (Invitrogen; Thermo Fisher Scientific, Inc.) according to the manufacturer's recommendations. miRNA complementary DNA (cDNA) was converted from total RNA by using a PrimeScript miRNA cDNA Synthesis Kit (cat. no. D350A; Takara Biotechnology Co., Ltd.). miR-193b quantification was performed using specific primers and probes using TaqMan MicroRNA Assays (cat. no. 4426961; Applied Biosystems, Thermo Fisher Scientific, Inc.); RNA U6 was used as the internal control. The primer sequences for U6 were: Forward: 5'-GTGCTCGCT TCGGCAGCACATATAC-3' and reverse: 5'-AAAAATATG

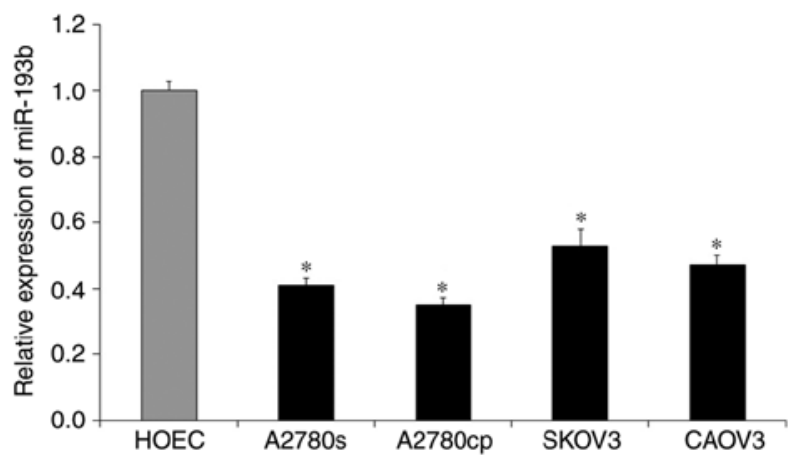

Figure 1. The relative expression of miR-193b in human ovarian cancer cells. The expression of miR-193b was measured using reverse transcriptionquantitative-PCR assay. The relative expression was normalized with U6 and the data were presented as the mean \pm standard deviation. ${ }^{*} \mathrm{P}<0.05$. $\mathrm{miR}$, microRNA.

GAACGCTCACGAATTTG-3'. Relative mRNA expression was calculated by using the $2^{-\Delta \Delta \mathrm{Cq}}$ method.

Bioinformatics analysis. To identify the potential target of miR-193b, genes were predicted by searching targetscan (http://www.targetscan.org) and mirbase targets (http: //microrna. sanger.ac.uk/cgi-bin/targets/v5/search.pl). STMN1 was predicted to be a target of miR-193b.

Luciferase reporter assay. A fragment from the 3'UTR of STMN1 gene containing the predicted binding site of miR-193b was amplified using PCR from genomic DNA. The amplified fragment was cloned into the UTR downstream of the luciferase gene in the pMIR-reporter luciferase vector (Ambion; Thermo Fisher Scientific, Inc.). A corresponding mutant construct was used as the control. Ovarian cancer cells were co-transfected with the testing firefly luciferase reporter plasmid together with a Renilla luciferase plasmid. Dualluciferase activities were measured by using a Dual-Glo Luciferase Assay System (Promega Corporation). Results were presented as the ratio between the treatment and the control group.

Western blot assay. Total protein was extracted by using RIPA lysis buffer (Beyotime Institute of Biotechnology) according to the manufacturer's recommendations. Extracted proteins were separated on a $10 \%$ SDS-PAGE gel and transferred to a PVDF membrane (GE Healthcare). The PVDF membrane was then blocked with 5\% BSA for $2 \mathrm{~h}$ at RT and incubated with antibodies against STMN1 (cat. no. 3352; Cell Signaling Technology, Inc.) and GAPDH (cat. no. 2118; Cell Signaling Technology, Inc.) at a 1:1,000 dilution for $2 \mathrm{~h}$ at RT. The membrane was then washed and further incubated with the secondary antibody (cat. no. 7074; Cell Signaling Technology, Inc.) at a 1:1,000 dilution for $2 \mathrm{~h}$ at RT. The bands were then detected by using the Novex ECL HRP chemiluminescent substrate reagent kit (Invitrogen; Thermo Fisher Scientific, Inc.).

Statistical analysis. All data were analyzed using a one-way ANOVA and Tukey's HSD test (post hoc test). Statistical analyses were performed using SPSS Statistics v19. The data 



Figure 2. miR-193b inhibits ovarian cancer cell growth. (A) The expression of miR-193b in ovarian cancer cells was measured using RT-qPCR assay. (B) The expression of miR-193b in transfected ovarian cancer cells was measured using RT-qPCR assay. (C) The upregulation of miR-193b significantly inhibited A2780s cell proliferation. (D) The upregulation of miR-193b significantly inhibited A2780cp cell proliferation. (E) The upregulation of miR-193b significantly inhibited CAOV3 cell proliferation. (F) The downregulation of miR-193b significantly induced HOEC cell proliferation. The data were presented as the mean \pm standard deviation. ${ }^{*} \mathrm{P}<0.05$. miR, microRNA; NC, negative control; OD, optical density; RT-q, reverse transcription-quantitative.

were expressed as the mean \pm standard deviation $(\mathrm{SD}) . \mathrm{P}<0.05$ was considered to indicate a statistically significant difference. All experiments were performed in triplicate.

\section{Results}

miR-193b is down-regulated in ovarian cancer cells. To investigate the biological roles of miR-193b in ovarian cancer, the expression level of miR-193b in a number of ovarian cancer cell lines was measured by using RT-qPCR assay. As presented in Fig. 1, the expression of miR-193b in ovarian cancer cells (A2780cp, A2780s, SKOV3 and CAOV3) was significantly down-regulated compared with the non-malignant HOEC cell line (all, $\mathrm{P}<0.05$; Fig. 1). These results suggested that the down-regulation of miR-193b may be associated with the development of ovarian cancer.

Effects of miR-193b transfection on ovarian cancer cell proliferation. To investigate the effects of miR-193b on ovarian cancer cell proliferation, a CCK-8 assay was performed. RT-qPCR results indicated miR-193b mimic or inhibitor transfection significantly regulated the expression of miR-193b in ovarian cancer cells $(\mathrm{P}<0.05$; Fig. $2 \mathrm{~A}$ and $\mathrm{B})$. CCK-8 assay results demonstrated that the up-regulation of miR-193b inhibited the proliferation of A2780s ( $\mathrm{P}<0.05$; Fig. $2 \mathrm{C}), \mathrm{A} 2780 \mathrm{cp}(\mathrm{P}<0.05$; Fig. 2D) and CAOV3 (P<0.05; Fig. 2E) compared with mock cells. In parallel, the miR-193b inhibitor or inhibitor control was transfected into HOEC cells to investigate the effects of miR-193b in normal ovary epithelial cell. As presented in Fig. 2F, the down-regulation of miR-193b increased HOEC cell proliferation $(\mathrm{P}<0.05)$. The miR-NC and inhibitor control transfection was not indicated to affect ovarian cancer cell proliferation. Therefore, miR-193b inhibited the on ovarian cancer cell proliferation in vitro.

Effects of miR-193b transfection on ovarian cancer cell apoptosis. To investigate whether miR-193b regulated ovarian cancer cell proliferation through the induction of cell 


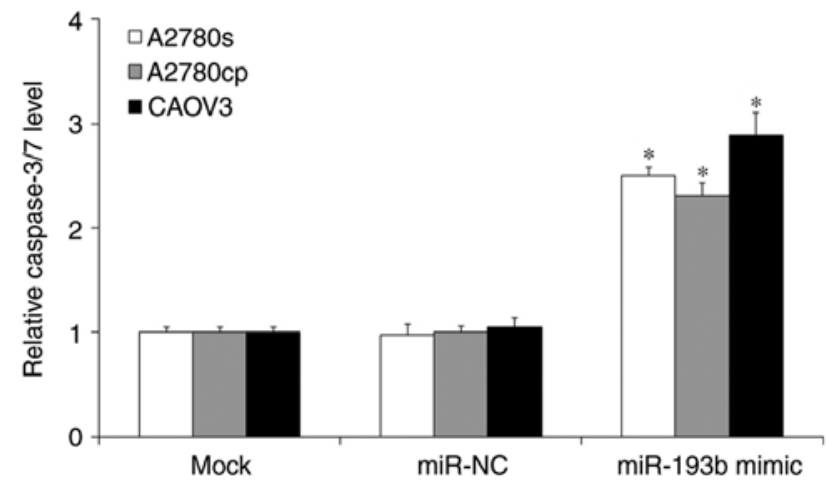

Figure 3. The caspase-3/7 activity in mock and transfected human ovarian cancer cells. The data were presented as the mean \pm standard deviation. ${ }^{*} \mathrm{P}<0.05$. miR, microRNA; NC, negative control.

apoptosis, a cell apoptosis assay was performed. As presented in Fig. 3, the induction of miR-193b significantly induced ovarian cancer cell apoptosis compared with the mock ovarian cancer cells ( $\mathrm{P}<0.05$; Fig. 3$)$, and the miR-NC transfection did not affect ovarian cancer cell apoptosis ( $\mathrm{P}>0.05$; Fig. 3).

STMN1 is a direct target of $\mathrm{miR}-193 \mathrm{~b}$. The results of the current study indicated that miR-193b regulated ovarian cancer cell proliferation and apoptosis, therefore, the present study aimed to determine the downstream target of miR-193b in ovarian cancer cells. By using prediction programs, STMN1 was indicated as a potential target of miR-193b (Fig. 4A). Western blot results showed that the expression of STMN1 was significantly increased in ovarian cancer cells compared with the non-malignant HOEC cell line (Fig. 5A). To confirm whether STMN1 is a direct target of miR-193b in ovarian cancer cells, miR-193b and luciferase reporter plasmids were co-transfected with wild type (WT) or mutant type (Mut) 3'-UTRs of STMN1, into ovarian cancer cells, and the luciferase value was measured. As presented in Fig. 4B, miR-193b remarkably decreased the luciferase activity in the WT group but not in the Mut group in ovarian cancer cells. The results suggested that STMN1 is a direct target of miR-193b in ovarian cancer cells.

miR-193b modulated ovarian cancer cell growth through targeting STMN1. To investigate whether miR-193b regulated ovarian cancer cell growth through targeting STMN1, a western blot assay was performed. Ovarian cancer cells were transfected with miR-193b mimic or miR-NC and cultured for $48 \mathrm{~h}$, and the expression of STMN1 was determined. The results indicated that the up-regulation of miR-193b decreased the expression of STMN1 in ovarian cancer cells, while miR-NC transfection did not affect the expression of STMN1 in ovarian cancer cells (Fig. 5B). These results suggested that miR-193b modulated ovarian cancer cell growth through targeting STMN1.

\section{Discussion}

Ovarian cancer exhibits the eighth highest mortality rate in women worldwide (1). Despite the low prevalence rate, the majority of patients exhibit cancer recurrence and cancer-associated mortality (16). Therefore, the investigation into the
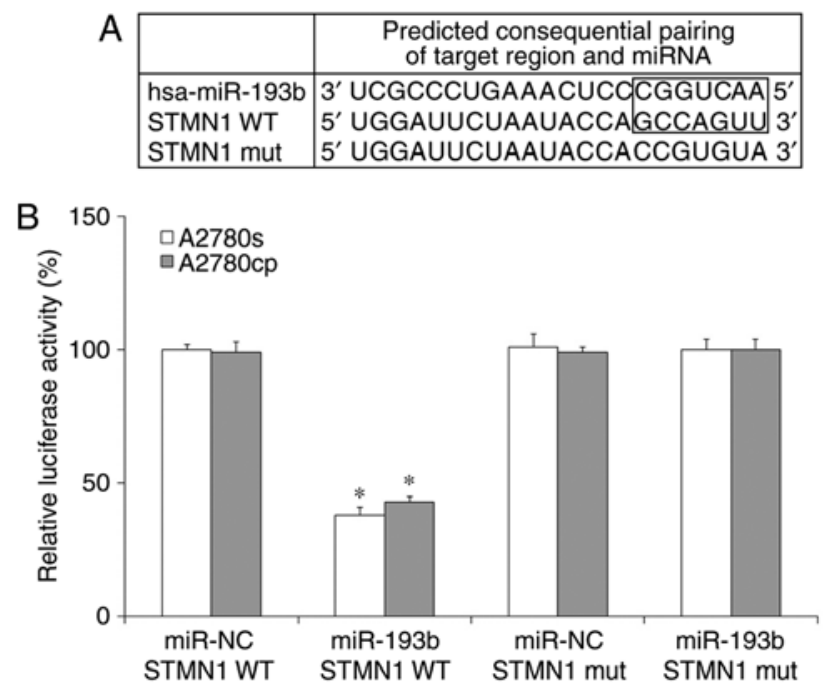

Figure 4. miR-193b directly targets STMN1 in human ovarian cancer cells. (A) The wild-type and mutant miR-193b targeting sequences in STMN1 mRNA. (B) The luciferase activity in human ovarian cancer cells. The data were presented as the mean \pm standard deviation. ${ }^{*} \mathrm{P}<0.05$. miR, microRNA; STMN1, stathmin 1; mut, mutant; WT, wild-type.
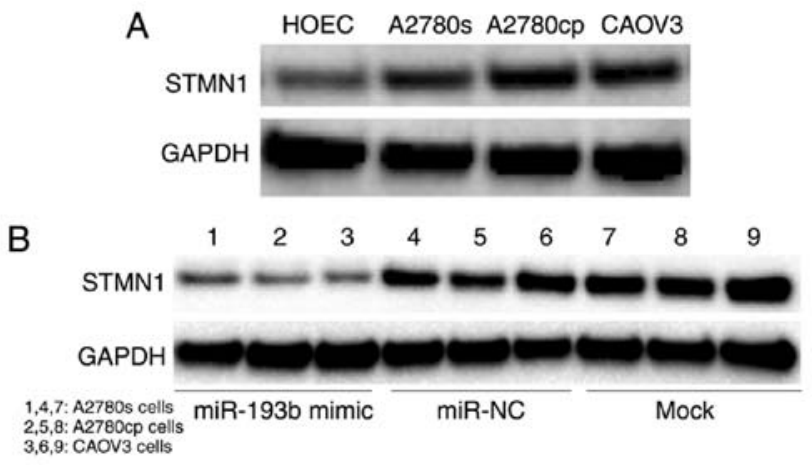

Figure 5. miR-193b regulates STMN1 expression in human ovarian cancer cells. (A) The STMN1 expression in human ovarian cancer cells and the non-malignant cells. (B) Upregulation of miR-193b decreased the expression of STMN1 expression in human ovarian cancer cells. miR, microRNA; STMN1, stathmin 1; NC, negative control.

mechanisms underlying the pathogenesis of ovarian cancer is urgently required. A number of studies have indicated that miRNAs are associated in the pathogenesis of human cancers, indicating that they contributed to the cancer cell proliferation, differentiation, cell cycle and apoptosis and also serve as oncogenes and tumor suppressors (17-19). Recent studies have indicated that miR-193b is dysregulated and serves as a tumor suppressor in patients exhibiting a variety of primary tumors, including breast cancer, gastric cancer, cervical cancer, prostate cancer and ovarian cancer (11-15,20-23). Wu et al reported that the expression of miR-193b was significantly down-regulated in endometrioid adenocarcinoma (24). Rauhala et al reported that miR-193b is a tumor suppressor in prostate cancer (25). Chen et al revealed that miR-193b was significantly down-regulated in melanoma tissues, and the up-regulation of miR-193b decreased cell proliferation by regulating cyclin D1 (26). Li et al indicated that miR-193b was a novel biomarker for patients with ovarian cancer (27). 
However, the role of miR-193b in ovarian cancer is yet to be determined.

In the present study, miR-193b was demonstrated to be down-regulated in human ovarian cancer cells compared with non-malignant cells. Therefore, it was hypothesized that miR-193b may act as a tumor suppressor in the development of ovarian cancer. The current study indicated that the up-regulation of miR-193b inhibited ovarian cancer proliferation and induced ovarian cancer apoptosis. The results also revealed that the down-regulation of miR-193b increased the non-malignant HOEC cell proliferation. Further study indicated that STMN1 is a direct target of miR-193b in human ovarian cancer cell. STMN1, which is also known as metablastin and oncoprotein 18 , is a protein that is encoded by the STMN1 gene. STMN1 is highly conserved and is important for the regulation of the cell cytoskeleton (28). STMN1 encode for s a cytosolic phosphoprotein, and this protein serves an important role in the function of the mitotic spindle (29). Therefore, it can be suggested that STMN1 serves an important role in the regulation of cell growth (30). Previous studies have indicated that STMN1 is over-expressed in many types of human cancer types, including in hepatocellular cancer, gastric cancer, breast cancer and prostate cancer (31-34). STMN1 has also been suggested to be an an oncogene in human cancer. In the current study, the expression of STMN1 was indicated to be up-regulated in human ovarian cancer cells, and the up-regulation of miR-193b significantly decreased the expression of STMN1 in human ovarian cancer cells. Based on these findings, it can be suggested that the up-regulation of miR-193b effectively regulated ovarian cancer cell growth, at least in part, through the inhibition of STMN1 expression. However, ovarian tumorigenesis is a complicated process and a lot of other factors are involved within this, and whether STMN1 is the key targeting gene of miR-193b requires further investigation.

In conclusion, our study revealed that miR-193b was down-regulated in human ovarian cancer cells and the regulation of miR-193b regulated human ovarian cancer cell growth through targeting STMN1. These results provide novel information into the molecular mechanism underlying the effects of miR-193b on human ovarian cancer cells, and suggest that the regulation of miR-193b may benefit ovarian cancer treatment in the future.

\section{Acknowledgements}

Not applicable.

\section{Funding}

No funding was received.

\section{Availability of data and materials}

The datasets used and analyzed during the current study are available from the corresponding author on reasonable request.

\section{Authors' contributions}

HL designed the study; HL, YX and DZ performed the experiments, analyzed the data and prepared the manuscript. HL reviewed the manuscript. All authors read and approved the final manuscript.

\section{Ethics approval and consent to participate}

Not applicable.

\section{Patient consent for publication}

Not applicable.

\section{Competing interests}

The authors declare that they have no competing interests.

\section{References}

1. Bray F, Ferlay J, Soerjomataram I, Siegel RL, Torre LA and Jemal A: Global cancer statistics 2018: GLOBOCAN estimates of incidence and mortality worldwide for 36 cancers in 185 countries. CA Cancer J Clin 68: 394-424, 2018.

2. Vaughan S, Coward JI, Bast RC Jr, Berchuck A, Berek JS, Brenton JD, Coukos G, Crum CC, Drapkin R, Etemadmoghadam D, et al: Rethinking ovarian cancer: Recommendations for improving outcomes. Nat Rev Cancer 11: 719-725, 2011.

3. Weidle UH, Dickopf S, Hintermair C, Kollmorgen G, Birzele F and Brinkmann U: The role of micro RNAs in breast cancer metastasis: Preclinical validation and potential therapeutic targets. Cancer Genomics Proteomics 15: 17-39, 2018.

4. Jiang YW and Chen LA: microRNAs as tumor inhibitors, oncogenes, biomarkers for drug efficacy and outcome predictors in lung cancer (review). Mol Med Rep 5: 890-894, 2012.

5. Kelly BD, Miller N, Healy NA, Walsh K and Kerin MJ: A review of expression profiling of circulating microRNAs in men with prostate cancer. BJU Int 111: 3-4, 2013.

6. Nair VS, Maeda LS and Ioannidis JP: Clinical outcome prediction by microRNAs in human cancer: A systematic review. J Natl Cancer Inst 104: 528-540, 2012.

7. Iorio MV and Croce CM: MicroRNAs in cancer: Small molecules with a huge impact. J Clin Oncol 27: 5848-5856, 2009.

8. Glud M, Rossing M, Hother C, Holst L, Hastrup N, Nielsen FC, Gniadecki R and Drzewiecki KT: Downregulation of miR-125b in metastatic cutaneous malignant melanoma. Melanoma Res 20: 479-484, 2010

9. Bushati $\mathrm{N}$ and Cohen SM: microRNA functions. Annu Rev Cell Dev Biol 23: 175-205, 2007.

10. Croce CM: Causes and consequences of microRNA dysregulation in cancer. Nat Rev Genet 10: 704-714, 2009.

11. Yin W, Nie Y, Chen L, Wang Q, Liu S, He X and Wang W: Deregulation of microRNA-193b affects the proliferation of liver cancer via myeloid cell leukemia-1. Oncol Lett 15: 2781-2788, 2018.

12. $\mathrm{Wu} \mathrm{H}$ and Zhou $\mathrm{C}$ : Long non-coding RNA UCA1 promotes lung cancer cell proliferation and migration via microRNA-193a/HMGB1 axis. Biochem Biophys Res Commun 496: 738-745, 2018.

13. Fang C, Dai CY, Mei Z, Jiang MJ, Gu DN, Huang Q and Tian L: microRNA-193a stimulates pancreatic cancer cell repopulation and metastasis through modulating TGF- $\beta 2 /$ TGF- $\beta$ RIII signalings. J Exp Clin Cancer Res 37: 25, 2018.

14. Chan CM, Lai KKY, Ng EKO, Kiang MN, Kwok TWH, Wang HK, Chan KW, Law TT, Tong DK, Chan KT, et al: Serum microRNA-193b as a promising biomarker for prediction of chemoradiation sensitivity in esophageal squamous cell carcinoma patients. Oncol Lett 15: 3273-3280, 2018.

15. Han D, Wang J and Cheng G: LncRNA NEAT1 enhances the radio-resistance of cervical cancer via miR-193b-3p/CCND1 axis. Oncotarget 9: 2395-2409, 2017.

16. Chan JK, Cheung MK, Husain A, Teng NN, West D, Whittemore AS, Berek JS and Osann K: Patterns and progress in ovarian cancer over 14 years. Obstet Gynecol 108: 521-528, 2006.

17. Fabbri M, Ivan M, Cimmino A, Negrini $M$ and Calin GA: Regulatory mechanisms of microRNAs involvement in cancer. Expert Opin Biol Ther 7: 1009-1019, 2007. 
18. Croce CM and Calin GA: miRNAs, cancer, and stem cell division. Cell 122: 6-7, 2005.

19. Brown JR and Sanseau P: A computational view of microRNAs and their targets. Drug Discov Today 10: 595-601, 2005.

20. Tahiri A, Leivonen SK, Lüders T, Steinfeld I, Ragle Aure M, Geisler J, Mäkelä R, Nord S, Riis ML, Yakhini Z, et al: Deregulation of cancer-related miRNAs is a common event in both benign and malignant human breast tumors. Carcinogenesis 35 76-85, 2014.

21. Zhou H, Wang $\mathrm{K}, \mathrm{Hu} \mathrm{Z}$ and Wen J: TGF- $\beta 1$ alters microRNA profile in human gastric cancer cells. Chin J Cancer Res 25 102-111, 2013.

22. Cheung TH, Man KN, Yu MY, Yim SF, Siu NS, Lo KW, Doran G, Wong RR, Wang VW, Smith DI, et al: Dysregulated microRNAs in the pathogenesis and progression of cervical neoplasm. Cell Cycle 11: 2876-2884, 2012.

23. Xie C, Jiang XH, Zhang JT, Sun TT, Dong JD, Sanders AJ, Diao RY, Wang Y, Fok KL, Tsang LL, et al: CFTR suppresses tumor progression through miR-193b targeting urokinase plasminogen activator ( $\mathrm{UPA}$ ) in prostate cancer. Oncogene 32 : 2282-2291, 2013.

24. Wu W, Lin Z, Zhuang Z and Liang X: Expression profile of mammalian microRNAs in endometrioid adenocarcinoma. Eur J Cancer Prev 18: 50-55, 2009.

25. Rauhala HE, Jalava SE, Isotalo J, Bracken $H$, Lehmusvaara $S$, Tammela TL, Oja H and Visakorpi T: miR-193b is an epigenetically regulated putative tumor suppressor in prostate cancer. Int J Cancer 127: 1363-1372, 2010.
26. Chen J, Feilotter HE, Paré GC, Zhang X, Pemberton JG, Garady C, Lai D, Yang X and Tron VA: MicroRNA-193b represses cell proliferation and regulates cyclin D1 in melanoma. Am J Pathol 176: 2520-2529, 2010.

27. Li H, Xu Y, Qiu W, Zhao D and Zhang Y: Tissue miR-193b as a novel biomarker for patients with ovarian cancer. Med Sci Monit 21: 3929-3934, 2015.

28. Kueh HY and Mitchison TJ: Structural plasticity in actin and tubulin polymer dynamics. Science 325: 960-963, 2009.

29. Rana S, Maples PB, Senzer N and Nemunaitis J: Stathmin 1: A novel therapeutic target for anticancer activity. Expert Rev Anticancer Ther 8: 1461-1470, 2008.

30. Rubin CI and Atweh GF: The role of stathmin in the regulation of the cell cycle. J Cell Biochem 93: 242-250, 2004.

31. Belletti B and Baldassarre G: Stathmin: A protein with many tasks. New biomarker and potential target in cancer. Expert Opin Ther Targets 15: 1249-1266, 2011.

32. Mistry SJ and Atweh GF: Role of stathmin in the regulation of the mitotic spindle: Potential applications in cancer therapy. Mt Sinai J Med 69: 299-304, 2002.

33. Ke B, Wu LL, Liu N, Zhang RP, Wang CL and Liang $\mathrm{H}$ : Overexpression of stathmin 1 is associated with poor prognosis of patients with gastric cancer. Tumour Biol 34: 3137-3145, 2013.

34. Tamura K, Yoshie M, Miyajima E, Kano M and Tachikawa E: Stathmin regulates hypoxia-inducible factor- $1 \alpha$ expression through the mammalian target of rapamycin pathway in ovarian clear cell adenocarcinoma. ISRN Pharmacol 2013: 279593, 2013. 